\title{
Another Criterion For The Riemann Hypothesis
}

\author{
Frank Vega
}

CopSonic, 1471 Route de Saint-Nauphary 82000 Montauban, France

\begin{abstract}
Let's define $\delta(x)=\left(\sum_{q \leq x} \frac{1}{q}-\log \log x-B\right)$, where $B \approx 0.2614972128$ is the Meissel-Mertens constant. The Robin theorem states that $\delta(x)$ changes sign infinitely often. For $x \geq 2$, Nicolas defined the function $u(x)=\sum_{q>x}\left(\log \left(\frac{q}{q-1}\right)-\frac{1}{q}\right)$ and proved that $0<u(x) \leq \frac{1}{2 \times(x-1)}$. We define the another function $\varpi(x)=\left(\sum_{q \leq x} \frac{1}{q}-\log \log \theta(x)-B\right)$, where $\theta(x)$ is the Chebyshev function. Using the Nicolas theorem, we demonstrate that the Riemann Hypothesis is true if and only if the inequality $\varpi(x)>u(x)$ is satisfied for all number $x \geq 3$. Consequently, we show that when the inequality $\varpi(x) \leq 0$ is satisfied for some number $x \geq 3$, then the Riemann Hypothesis should be false. Moreover, if the inequalities $\delta(x) \leq 0$ and $\theta(x) \geq x$ are satisfied for some number $x \geq 3$, then the Riemann Hypothesis should be false. In addition, we know that $\lim _{x \rightarrow \infty} \varpi(x)=0$ because of $\lim _{x \rightarrow \infty} \delta(x)=0$ and $\lim _{x \rightarrow \infty} \frac{\theta(x)}{x}=1$.
\end{abstract}

Keywords: Riemann hypothesis, Nicolas theorem, Chebyshev function, prime numbers 2000 MSC: 11M26, 11A41, 11A25

\section{Introduction}

In mathematics, the Riemann Hypothesis is a conjecture that the Riemann zeta function has its zeros only at the negative even integers and complex numbers with real part $\frac{1}{2}$ [1]. Let $N_{n}=$ $2 \times 3 \times 5 \times 7 \times 11 \times \cdots \times p_{n}$ denotes a primorial number of order $n$ such that $p_{n}$ is the $n^{\text {th }}$ prime number. Say Nicolas $\left(p_{n}\right)$ holds provided

$$
\prod_{q \mid N_{n}} \frac{q}{q-1}>e^{\gamma} \times \log \log N_{n} .
$$

The constant $\gamma \approx 0.57721$ is the Euler-Mascheroni constant, log is the natural logarithm, and $q \mid N_{n}$ means the prime number $q$ divides to $N_{n}$. The importance of this property is:

Theorem 1.1. [2], [3]. Nicolas $\left(p_{n}\right)$ holds for all prime number $p_{n}>2$ if and only if the Riemann Hypothesis is true.

In mathematics, the Chebyshev function $\theta(x)$ is given by

$$
\theta(x)=\sum_{p \leq x} \log p
$$

where $p \leq x$ means all the prime numbers $p$ that are less than or equal to $x$. We know this:

Email address: vega.frank@gmail.com (Frank Vega) 
Theorem 1.2. [4].

$$
\lim _{x \rightarrow \infty} \frac{\theta(x)}{x}=1
$$

Let's define $H=\gamma-B$ such that $B \approx 0.2614972128$ is the Meissel-Mertens constant [5]. We know from the constant $H$, the following formula:

Theorem 1.3. [6].

$$
\sum_{q}\left(\log \left(\frac{q}{q-1}\right)-\frac{1}{q}\right)=\gamma-B=H .
$$

For $x \geq 2$, Nicolas defined the function $u(x)$ as follows

$$
u(x)=\sum_{q>x}\left(\log \left(\frac{q}{q-1}\right)-\frac{1}{q}\right) .
$$

Nicolas showed that

Theorem 1.4. [3]. For $x \geq 2$ :

$$
0<u(x) \leq \frac{1}{2 \times(x-1)}
$$

Let's define:

$$
\delta(x)=\left(\sum_{q \leq x} \frac{1}{q}-\log \log x-B\right) .
$$

Robin theorem states the following result:

Theorem 1.5. [7]. $\delta(x)$ changes sign infinitely often.

In addition, the Mertens second theorem states that:

Theorem 1.6. [5].

$$
\lim _{x \rightarrow \infty} \delta(x)=0
$$

We define another function:

$$
\varpi(x)=\left(\sum_{q \leq x} \frac{1}{q}-\log \log \theta(x)-B\right) .
$$

Putting all together yields the proof that the inequality $\varpi(x)>u(x)$ is satisfied for a number $x \geq 3$ if and only if Nicolas $(p)$ holds, where $p$ is the greatest prime number such that $p \leq x$. In this way, we introduce another criterion for the Riemann Hypothesis based on the Nicolas criterion.

\section{Results}

Theorem 2.1. The inequality $\varpi(x)>u(x)$ is satisfied for a number $x \geq 3$ if and only if Nicolas $(p)$ holds, where $p$ is the greatest prime number such that $p \leq x$. 
Proof. We start from the inequality:

$$
\varpi(x)>u(x)
$$

which is equivalent to

$$
\left(\sum_{q \leq x} \frac{1}{q}-\log \log \theta(x)-B\right)>\sum_{q>x}\left(\log \left(\frac{q}{q-1}\right)-\frac{1}{q}\right) .
$$

Let's add the following formula to the both sides of the inequality,

$$
\sum_{q \leq x}\left(\log \left(\frac{q}{q-1}\right)-\frac{1}{q}\right)
$$

and due to the theorem 1.3 , we obtain that

$$
\sum_{q \leq x} \log \left(\frac{q}{q-1}\right)-\log \log \theta(x)-B>H
$$

because of

$$
H=\sum_{q \leq x}\left(\log \left(\frac{q}{q-1}\right)-\frac{1}{q}\right)+\sum_{q>x}\left(\log \left(\frac{q}{q-1}\right)-\frac{1}{q}\right)
$$

and

$$
\sum_{q \leq x} \log \left(\frac{q}{q-1}\right)=\sum_{q \leq x} \frac{1}{q}+\sum_{q \leq x}\left(\log \left(\frac{q}{q-1}\right)-\frac{1}{q}\right) .
$$

Let's distribute it and remove $B$ from the both sides:

$$
\sum_{q \leq x} \log \left(\frac{q}{q-1}\right)>\gamma+\log \log \theta(x)
$$

since $H=\gamma-B$. If we apply the exponentiation to the both sides of the inequality, then we have that

$$
\prod_{q \leq x} \frac{q}{q-1}>e^{\gamma} \times \log \theta(x)
$$

which means that Nicolas $(p)$ holds, where $p$ is the greatest prime number such that $p \leq x$. The same happens in the reverse implication.

Theorem 2.2. The Riemann Hypothesis is true if and only if the inequality $\varpi(x)>u(x)$ is satisfied for all number $x \geq 3$.

Proof. This is a direct consequence of theorems 1.1 and 2.1.

Lemma 2.3. If the inequality $\varpi(x) \leq 0$ is satisfied for some number $x \geq 3$, then the Riemann Hypothesis should be false.

Proof. This is an implication of theorems 1.4, 2.1 and 2.2.

Lemma 2.4. If the inequalities $\delta(x) \leq 0$ and $\theta(x) \geq x$ are satisfied for some number $x \geq 3$, then the Riemann Hypothesis should be false. 
Proof. If the inequalities $\delta(x) \leq 0$ and $\theta(x) \geq x$ are satisfied for some number $x \geq 3$, then we obtain that $\varpi(x) \leq 0$ is also satisfied, which means that the Riemann Hypothesis should be false according to the lemma 2.3 .

\section{Lemma 2.5.}

$$
\lim _{x \rightarrow \infty} \varpi(x)=0 .
$$

Proof. We know that $\lim _{x \rightarrow \infty} \varpi(x)=0$ for the limits $\lim _{x \rightarrow \infty} \delta(x)=0$ and $\lim _{x \rightarrow \infty} \frac{\theta(x)}{x}=1$. In this way, this is a consequence from the theorems 1.6 and 1.2.

\section{References}

[1] P. B. Borwein, S. Choi, B. Rooney, A. Weirathmueller, The Riemann Hypothesis: A Resource for the Afficionado and Virtuoso Alike, Vol. 27, Springer Science \& Business Media, 2008.

[2] J.-L. Nicolas, Petites valeurs de la fonction d'Euler et hypothese de Riemann, Séminaire de Théorie des nombres DPP, Paris 82 (1981) 207-218

[3] J.-L. Nicolas, Petites valeurs de la fonction d'Euler, Journal of number theory 17 (3) (1983) 375-388. doi:10.1016/0022-314X(83)90055-0.

[4] T. H. Grönwall, Some asymptotic expressions in the theory of numbers, Transactions of the American Mathematical Society 14 (1) (1913) 113-122. doi:10.2307/1988773.

[5] F. Mertens, Ein Beitrag zur analytischen Zahlentheorie., J. reine angew. Math. 1874 (78) (1874) $46-62$. doi:10.1515/crll.1874.78.46. URL https://doi.org/10.1515/crll.1874.78.46

[6] Y. Choie, N. Lichiardopol, P. Moree, P. Solé, On Robin's criterion for the Riemann hypothesis, Journal de Théorie des Nombres de Bordeaux 19 (2) (2007) 357-372. doi:10.5802/jtnb.591.

[7] G. Robin, Sur l'ordre maximum de la fonction somme des diviseurs, Séminaire Delange-Pisot-Poitou Paris 82 (1981) $233-242$. 